\title{
Epigenetics and Metabolism
}

\section{Contents}

9.1 Epigenetics and Metabolism - 180

9.2 Acetyl-Coenzyme A (Acetyl-CoA) - 180

9.2.1 Biosynthesis of Acetyl-CoA - 180

9.2.2 Acetyl-CoA as Cofactor of Histone Acetyltransferases - 183

9.3 Nicotinamide Adenine Dinucleotide (NAD) - 184

9.3.1 Biosynthesis of NAD - 185

9.3.2 NAD as Cofactor of Sirtuins and PARPs -187

9.4 S-adenosylmethionine (SAM) - 190

9.4.1 Biosynthesis of SAM - 190

9.4.2 SAM as Cofactor of DNA and Histone Methyltransferases - 191

9.5 Flavin Adenine Dinucleotide (FAD) - 194

9.5.1 Biosynthesis of FAD - 194

9.5.2 FAD as Cofactor of Lysine Demethylase 1 (LSD1) - 195

9.6 $\alpha$-Ketoglutarate $(\alpha K G)-196$

9.6.1 Biosynthesis of $\alpha$-Ketoglutarate - 196

9.6.2 $\alpha$ KG as Cofactor of TET-Family DNA Demethylases and Jumonji C-Family Histone Demethylases - 198

References - 200 


\section{What You Will Learn in This Chapter}

Most chromatin-modifying enzymes use metabolites as cofactors. Consequently, the cellular metabolism can influence the capacity of the cell to write or erase chromatin marks. This points to an intimate relationship between metabolic and epigenetic regulation. In this chapter, we describe the biosynthetic pathways of cofactors that are implicated in epigenetic and chromatin regulation and provide examples of how metabolic pathways can influence chromatin and epigenetic processes as well as their interplay in developmental and cancer biology.

\subsection{Epigenetics and Metabolism}

Cellular metabolism involves a set of complex and highly coordinated biochemical reactions that convert or use energy to maintain the living state of a cell. This process engages regulatory mechanisms that enable cells to sense nutrient availability and transmit the information through signaling networks. Metabolism impacts every cellular process. Nowadays, the study of metabolism is influencing all fields of biological research. Most chromatin-modifying enzymes use as cofactors important intermediates of cellular metabolism. Depending on dietary intake, metabolite concentrations can vary, and, in turn, can affect gene expression by modulating the activity of epigenetic pathways and associated chromatin modifying enzymes. Considering that the methylation of DNA and each post-translational modification (PTM) of proteins can be affected by many metabolic pathways, it is clear that the epigenome might act as a sensor of the whole metabolic network.

\subsection{Acetyl-Coenzyme A (Acetyl-CoA)}

Acetyl-coenzyme A (acetyl-CoA) is the cofactor of histone acetyltransferases (HATs) (- Fig. 9.1). The abundance of acetyl-CoA reflects the general energetic state of the cell. Biosynthesis, compartmentalization, and fluctuation of acetyl-CoA concentration change considerably in response to a series of physiological or pathological conditions. Thus, acetyl-CoA acts as metabolic sensor by signaling changes in metabolism that are then converted into gene expression states.

\subsubsection{Biosynthesis of Acetyl-CoA}

Acetyl-CoA comprises an acetyl moiety linked to coenzyme A, a derivative of vitamin B5 and cysteine (- Fig. 9.1). Acetyl-CoA is a central metabolite that interconnects multiple metabolic pathways. It can be found in two separate pools in the cell: the mitochondrial pool and the nuclear/cytosolic pool (• Fig. 9.2). This distinction is due to the fact that the inner mitochondrial membrane is impermeable to the highly charged acetyl-CoA molecule whereas nuclear pores allow acetyl-CoA to freely distribute between the cytosol and the nucleus. 


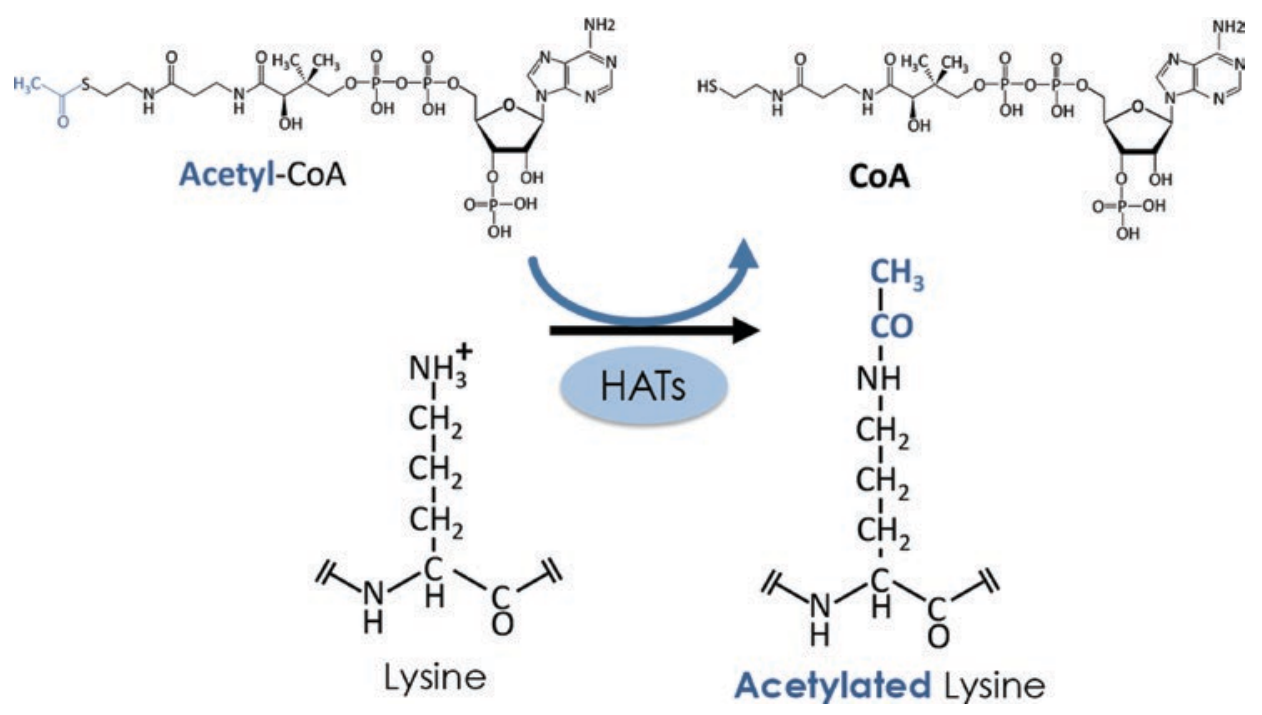

- Fig. 9.1 Acetyl-coenzyme A is the cofactor of histone acetyltransferases. Acetyl-coenzyme A (acetyl-CoA) comprises an acetyl moiety linked to coenzyme A, a derivative of vitamin B5 and cysteine. The schematic shows the acetylation of the $\varepsilon$-amino group of lysine mediated by histone acetyltransferases (HATs), whereby the acetyl group of acetyl-CoA is transferred to the lysine

The majority of acetyl-CoA is produced and consumed in mitochondria where it allows glycolytic ${ }^{1}$ pyruvate to enter the citric acid cycles, also known or tricarboxylic acid (TCA) cycle. In the cytosolic compartment, acetyl-CoA supports several anabolic reactions including synthesis of fatty acids and steroid and protein acetylation. In mitochondria, acetyl-CoA is produced through carboxylation of pyruvate to form acetyl-CoA, $\mathrm{CO}_{2}$, and $\mathrm{NADH}$, a reaction catalyzed by the mitochondrial pyruvate dehydrogenase complex (PDC) (• Fig. 9.2). At high glucose levels, acetyl-CoA is prevalently produced during the oxidation of glucose whereas at low glucose levels, it is generated as the end-product of the B-oxidation of fatty acids. Acetyl-CoA can also be produced by branched-chain amino acids, i.e., valine, leucine, and isoleucine, through cytosolic transamination to branched-chain $\alpha$-ketoacids followed by import into mitochondria. There, a reaction catalyzed by the mitochondrial branchedchain $\alpha$-ketoacid dehydrogenase generates NADH, acetyl-CoA, and other acyl-CoA thioesters. In addition to these nearly ubiquitous metabolic networks, mitochondrial acetyl-CoA can be produced by organ-specific pathways.

The nuclear-cytoplasmic level of acetyl-CoA in mammalian cells is established primarily by the combined action of two enzymes: ATP-citrate lyase (ACL) and acetyl-CoA synthetase (ACS) (• Fig. 9.2). ACL converts citrate into acetyl-CoA and oxaloacetate. This cytoplasmic pool of acetyl-CoA can originate from the

1 Glycolysis is a cytoplasmatic pathway that breaks down glucose into three carbon compounds and generates pyruvate and energy in form of ATP and NADH. 


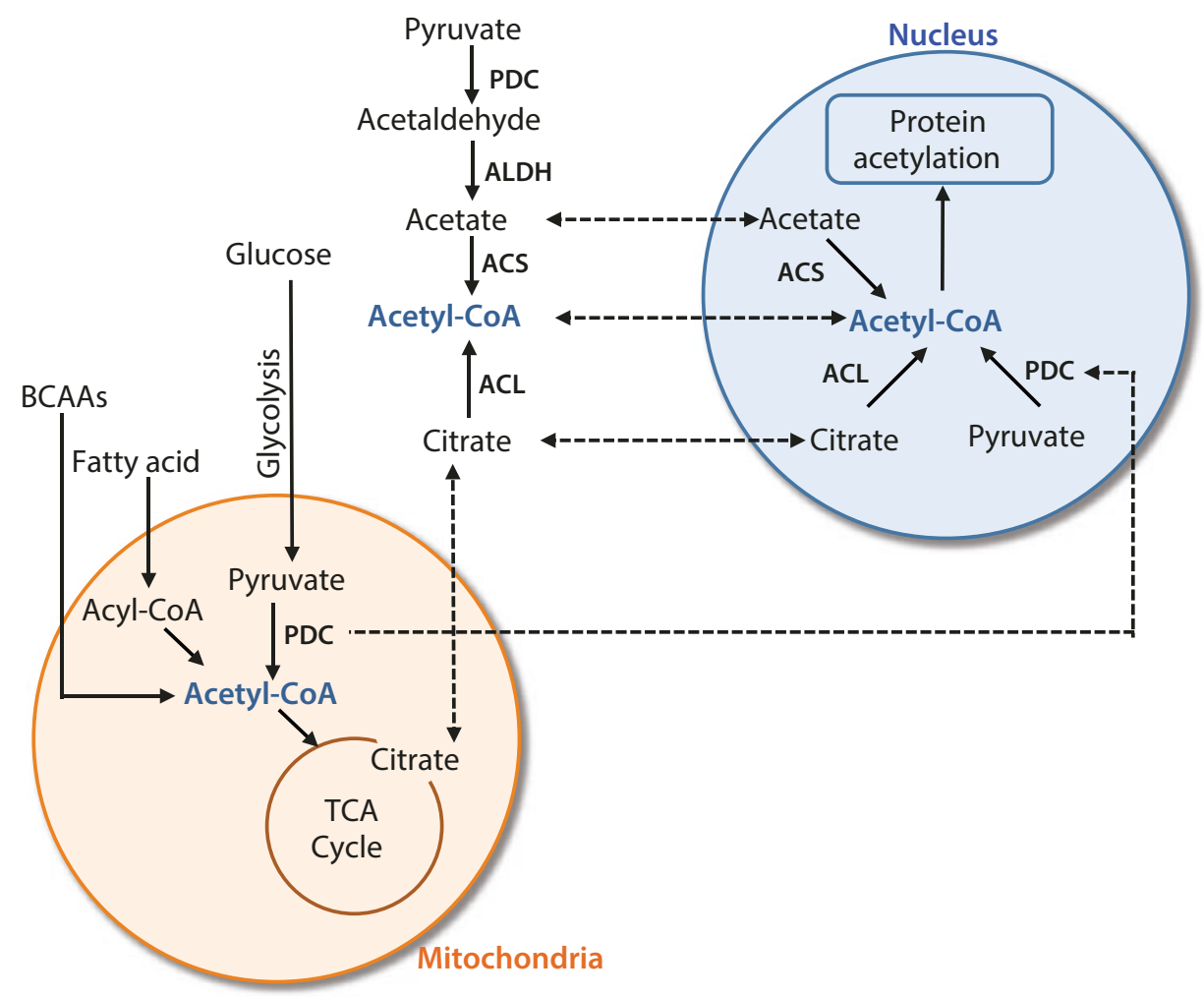

- Fig. 9.2 Metabolism of acetyl-CoA in mammalian cells. Acetyl-CoA can be found in two distinct pools in the cell: the mitochondrial pool and the nuclear/cytosolic pool. The majority of cellular acetyl$\mathrm{CoA}$ in mitochondria is generated and consumed in reactions involving the oxidative metabolism of glycolytic pyruvate, free fatty acids, branched-chain amino acids (BCAAs), or ketone bodies, which all converge on the citric acid cycle (TCA). In the cytosolic and nuclear compartment, acetyl-CoA is produced by ATP-citrate lyase (ACL) and acetyl-CoA synthetase (ACS). ACL converts citrate into oxaloacetate and acetyl-CoA. ACS utilizes acetate as a substrate for acetyl-CoA synthesis. These reactions support several anabolic reactions, including lipogenesis, steroidogenesis, and the synthesis of specific amino acids. PDC, ACL, and ACS are also found in the nucleus and produce acetyl-CoA therein. Their role in the nucleus mainly serves to produce acetyl-CoA for the acetylation of histones and non-histone proteins, thereby regulating gene expression

reductive carboxylation of glutamine. Cytosolic glutamine is transformed into glutamate, transported into mitochondria, and converted into $\alpha$-ketoglutarate, which generates citrate within the TCA cycle. Finally, citrate can be exported back to the cytosol where is converted into oxaloacetate and acetyl-CoA by ACL. ACS utilizes acetate as a substrate for acetyl-CoA synthesis. Production of acetyl-CoA within the nucleus is also possible due to the presence, in the nucleus, of ACL and ACS. In Saccharomyces cerevisiae, inactivation of ACS leads to rapid histone deacetylation and transcriptional defects, underscoring a direct link between acetyl-CoA producing enzymes, chromatin regulation, and gene expression. A novel pathway for nuclear acetyl-CoA synthesis has recently been suggested by showing that PDC can translocate from mitochondria to the nucleus upon growth factor stimulation (• Fig.9.2). 


\subsubsection{Acetyl-CoA as Cofactor of Histone Acetyltransferases}

Acetyl-CoA is the cofactor required by histone acetyltransferases (HATs), which attach an acetyl group to the $\varepsilon$-amino group of specific lysine residues ( $\bullet$ Fig. 9.1). Type B HATs are cytoplasmic enzymes, which modify free histones in the cytoplasm just after their synthesis ${ }^{2}$. Type A HATs are mainly nuclear enzymes and they are responsible for acetylation of histones and non-histone proteins in the nucleus and implicated in the epigenetic regulation of gene expression (see also book $>$ Chap. 1 of Wutz). In response to a series of physiological or pathological conditions, compartmentalization and fluctuation of acetyl-CoA concentration changes considerably and has a major impact in protein acetylation.

An important example of how acetyl-CoA synthesis responds to stimuli and affects histone acetylation and gene expression is provided by experiments studying growth of prototrophic ${ }^{3}$ strains of yeast under a nutrient-limiting environment. Under these conditions, cells enter into synchronous and highly robust oscillations of oxygen consumption, termed yeast metabolic cycles (YMCs). During YMCs, cells go through a synchronized transition between three metabolic phases, termed oxidative (OX), reductive and building (RB), and reductive and charging (RC). Acetyl-CoA levels strongly fluctuate as a function of the YMC. In the OX phase, mitochondrial respiration peaks and is associated with the rapid induction of genes involved in growth. In the RB phase, the rate of oxygen consumption begins to decrease and cell division initiates. In the RC phase, cells enter into a stationary and quiescent phase and express many genes that are negatively correlated with increasing growth rate. In the transition from the $\mathrm{RC}$ to the $\mathrm{OX}$ phase, cells re-enter into growth. This time point is characterized by a substantial increase in intracellular acetyl-CoA levels that trigger a series of histone acetylation events catalyzed by SptAda-Gcn5-acetyltransferase (SAGA, see also $>$ Chap. 2 of Paro) at genes important for growth, thereby enabling their rapid transcription and commitment to growth. The peak of acetyl-CoA at the OX/RB boundary is likely coordinated by induction of all enzymes required to convert ethanol (fermented via the consumption of glucose during the RB phase) into acetylaldehyde, into acetate, and, finally, into acetylCoA (Kaelin Jr. and McKnight 2013). Moreover, the link of this metabolic pathway with histone acetylation and gene expression is supported by the fact that acetylCoA synthase, which produces acetyl-CoA from acetate, is localized in the nucleus. In mammals, high acetyl-CoA concentrations also favor cell growth and replication at the expense of differentiation. The metabolism of embryonic stem cell (ESCs) shows the Warburg effect, a shift from oxidative phosphorylation to aerobic glycolysis that is also utilized by cancer cells ${ }^{4}$. Thus, ESCs produce acetyl-CoA and acetate

2 Note that the cytoplasmatic acetylation of newly synthesized histones plays a critical role in their transport and assembly into chromatin. Once incorporated into chromatin, the cytoplasmatic acetylation at these newly synthesized histone is removed by histone deacetylases.

3 Prototroph refers to an organisms or cell capable of synthesizing all its metabolites from inorganic material, without requiring organic nutrients.

4 When oxygen is limiting, cells can redirect the pyruvate generated by glycolysis away from mitochondrial oxidative phosphorylation by producing lactate (Warburg effect). This generation of lactate during anaerobic glycolysis allows glycolysis to continue, with consequent increase of Acetyl-CoA levels. 
through glycolysis, however, this processing step is rapidly downregulated during differentiation. This metabolic switch causes a loss of histone acetylation during the first hours of differentiation. The link of acetyl-CoA levels and pluripotency state is supported by experiments showing that an increase of acetate, a precursor of acetylCoA, delays ESC differentiation and blocks early histone deacetylation whereas glycolysis inhibition leads to deacetylation and differentiation of ESCs (Moussaieff et al. 2015). Acetyl-CoA fluctuations during ESC differentiation have also been correlated with changes in the expression of threonine dehydrogenase (TDH), which converts threonine into glycine and acetyl-CoA in mitochondria, with glycine facilitating one-carbon metabolism and the synthesis of S-adenosylmethionine (SAM) for DNA and histone methylation (see $>$ Sect. 9.4) and acetyl-CoA feeding the TCA cycle. Another example of the link between metabolism and epigenetic gene regulation are adipocytes, cells that are the primary constituent of adipose tissue and specialized in storing energy as fat. In adipocytes, ACL, the enzyme that converts glucose-derived citrate into acetyl-CoA, is required to increase histone acetylation in response to growth factor stimulation and during differentiation, indicating that glucose availability can affect histone acetylation in an ACL-dependent manner (Wellen et al. 2009).

The local abundance of acetyl-CoA within the nucleus might also affect HAT catalytic activity and regulate gene expression by controlling the levels of histone acetylation at specific genes. HATs display a relatively high dissociation constant (KD) for acetyl-CoA and consequently local changes in acetyl-CoA concentration might affect HAT activity. Studies in yeast have shown that acetyl-CoA levels $(3-30 \mu \mathrm{M})$ may fluctuate within a range to limit the activity of the HAT GCN5, which has a KD for acetyl-CoA of $8.5 \mathrm{mM}$. This is not the case for kinases, which use ATP as cofactor to phosphorylate, due to the elevated concentration of cellular ATP (roughly mM in cells) and their relatively high affinity for ATP (KD roughly $\mu \mathrm{M})$. It is also worth to mention that many HATs are subject to product inhibition by free CoA, indicating that the acetyl-CoA/CoA ratio might be the relevant regulator of HAT activity instead of the absolute levels of acetyl-CoA.

The concentration of acetyl-CoA can also alter the substrate specificity of HATs. The lysine acetyltransferases CBP and p300 acetylate many common lysine residues on histones $\mathrm{H} 3$ and $\mathrm{H} 4$. However, under limiting acetyl-CoA concentrations, CBP and p300 display distinct specificity and selectivity, with p300 showing the highest specificity for $\mathrm{H} 4 \mathrm{~K} 16$, that is $10^{18}$-fold higher than CBP.

\subsection{Nicotinamide Adenine Dinucleotide (NAD)}

Nicotinamide adenine dinucleotide (NAD) serves both as a critical cofactor for enzymes that catalyze reduction-oxidation reactions and as a co-substrate for three classes of enzymes: (i) the sirtuins (SIRTs), (ii) the adenosine diphosphate (ADP)ribose transferases (ARTs) and poly(ADP-ribose) polymerases (PARPs), and (iii) the cyclic ADP-ribose (cADPR) synthases (• Fig. 9.3) (Verdin 2015). 


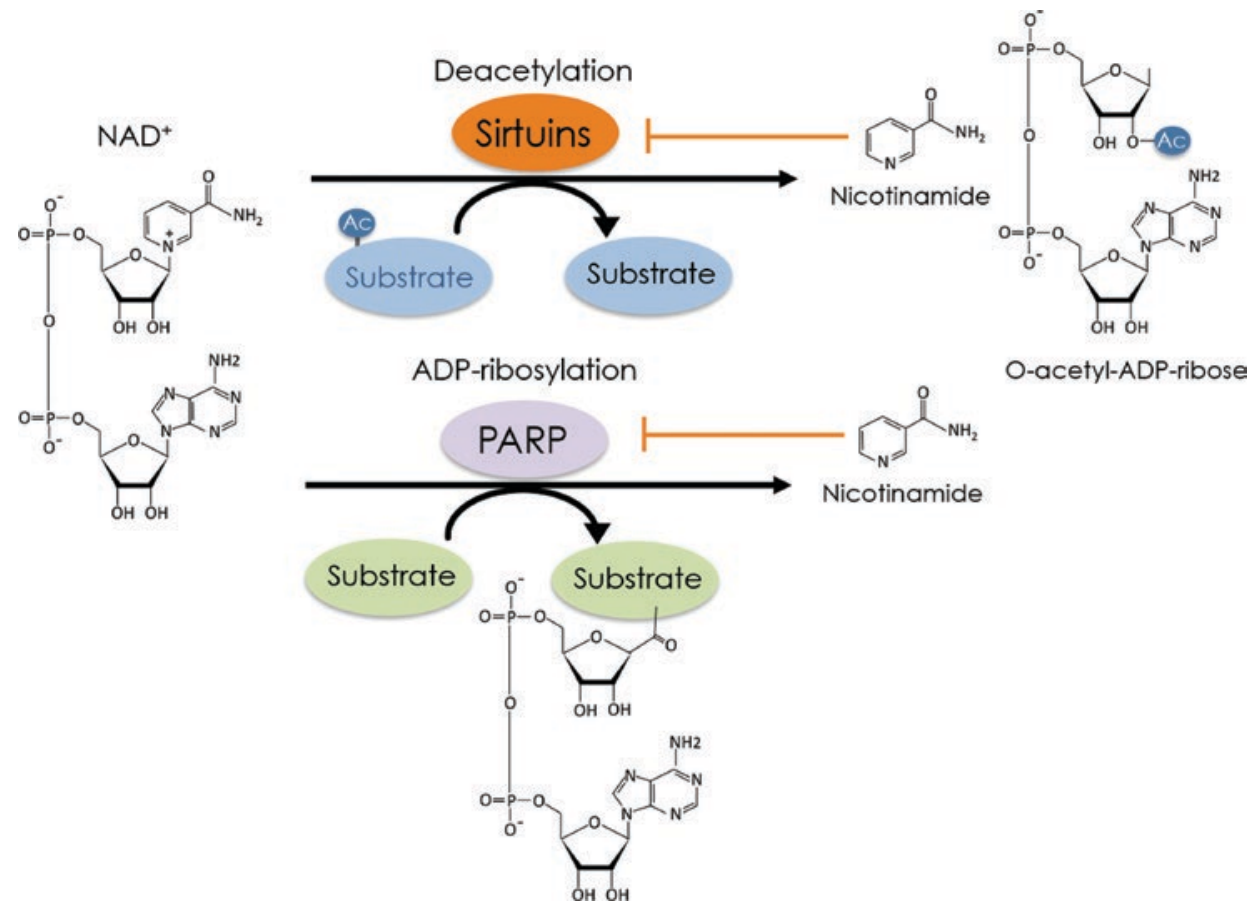

Fig. 9.3 Nicotinamide adenine dinucleotide (NAD) is a co-substrate for histone deacetylation and ADP-ribosylation. The structure of NAD in its oxidized form $\left(\mathrm{NAD}^{+}\right)$is shown. Sirtuins remove acetyl groups from target proteins. In this reaction, NAD is cleaved into nicotinamide, and the ADP-ribose serves as the acceptor for the removed acetyl group. Adenosine diphosphate (ADP)-ribose transferases (ARTs) and poly(ADP-ribose) polymerases (PARPs) transfer the ADP-ribose moiety from NAD to a target protein, releasing nicotinamide. Nicotinamide also serves as an inhibitory factor of SIRTs, ARTs, and PARPs

\subsubsection{Biosynthesis of NAD}

NAD can be synthesized from diverse dietary sources, including nicotinic acid (also known as Vitamin B3, an essential human nutrient) and nicotinamide, tryptophan, and nicotinamide riboside (NR). The major dietary source of NAD is nicotinic acid. NAD levels are maintained by three independent pathways: the Preiss-Handler pathway, the kinurenine pathway, and the NAD salvage pathway (• Fig. 9.4).

In the Preiss-Handler pathway, dietary nicotinic acid and the enzyme nicotinic acid phosphoribosyltransferase (NAPRT) generate nicotinic acid mononucleotide (NAMN), which is in turn converted by the nicotinamide mononucleotide adenylyltransferase (NMNAT) into nicotinic acid adenine dinucleotide (NAAD) in the presence of adenosine triphosphate (ATP). NMNAT has three forms with distinct subcellular localizations: NMNAT1 in the nucleus, NMNAT2 in the cytosol and Golgi, and NMNAT3 in the cytosol and mitochondria. The kinurenine pathway 


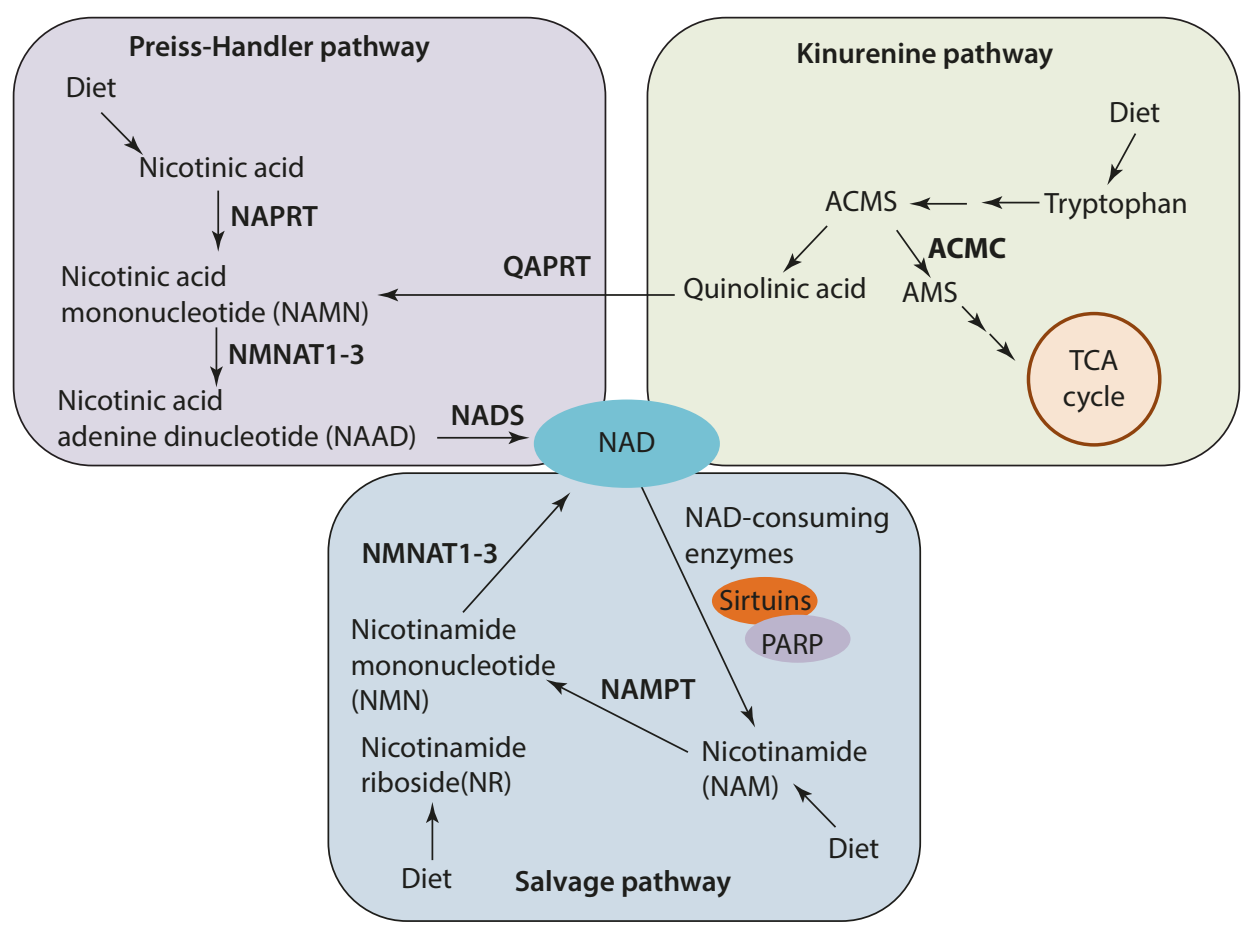

- Fig. 9.4 NAD biosynthetic pathways. Three independent pathways (Preiss-Handler, kinurenine and salvage pathways) maintain NAD levels. In the Preiss-Handler pathway, NAPRT enzyme transforms nicotinic acid into NAMN, which is then converted into NAAD by NMNAT1-3. NAD is generated from NAMN by the reaction catalyzed by NAD synthase (NADS). In the kinurenine pathway, NAD is produced from tryptophan. Spontaneous condensation ACMS and rearrangement into quinolinic acid make the kinurenine pathway to converge into the Preiss-Handler pathway for the production of NAD. The NAD salvage pathway recycles the nicotinamide generated as a by-product of the enzymatic activities of NAD-consuming enzymes SIRTs, ARTs, and PARPs. NAMPT uses nicotinamide to produce NMN, which is then converted into NAD via the different NMNATs as in the Preiss-Handler pathway. Nicotinic acid phosphoribosyltransferase (NAPRT), Nicotinic acid mononucleotide (NAMN), Nicotinic acid adenine dinucleotide (NAAD), Nicotinamide mononucleotide adenylyltransferase (NMNAT1-3), 2-amino-3-carboxymuconate semialdehyde (ACMS), Nicotinamide phosphoribosyltransferase (NAMPT), Nicotinamide mononucleotide (NMN)

produces NAD from tryptophan and converges into the Preiss-Handler pathway since 2-amino-3-carboxymuconate semialdehyde (ACMS) undergoes spontaneous condensation and rearranges into quinolinic acid, which is transformed into NAMN.

The NAD salvage pathway is a key pathway for maintaining cellular NAD levels since it recycles the nicotinamide generated as a by-product of the enzymatic activities of NAD-consuming enzymes, the SIRTs, ARTs, and PARPs. Nicotinamide phosphoribosyltransferase (NAMPT) recycles nicotinamide into nicotinamide mononucleotide (NMN), which is then converted into NAD via the different NMNATs as in the Preiss-Handler pathway. As it will be discussed in $>$ Sect. 9.3.2, nicotinamide also serves as an inhibitory factor of SIRTs, ARTs, and PARPs (• Fig. 9.3). Consequently, the NAD salvage pathway leads not only to recycling of nicotinamide into NAD but also relieves nicotinamide inhibition of NAD-consuming enzymes. 
Cellular NAD levels decline during the process of chronological aging and in progeroid $^{5}$ states. NAD levels decrease in animals kept under a high fat diet whereas NAD increases under exercise and caloric restriction. The relationship between NAD levels and the nutritional state of the organism has also been confirmed in studies of the circadian clock, which is encoded by a transcription-translation feedback loop that synchronizes behavior and metabolism with the light-dark cycle. Both the expression of NAMPT, the rate-limiting enzyme in the NAD salvage pathway, and the levels of NAD cycle with a 24-hour rhythm are regulated by the core clock machinery in mice.

NAMPT has been implicated in the decline of cellular NAD levels associated with aging. NAMPT expression decreases during age and forced expression of NAMPT delays senescence and substantially lengthens cell lifespan. In addition, lifelong muscle-specific Nampt transgene expression preserved muscle NAD levels and exercise capacity in aged mice (Frederick et al. 2016). The link between NAD levels and aging has initiated considerable efforts to manipulate NAD concentrations in therapies aimed at disease prevention and life-span extension. Pharmacological approaches able to improve NAD availability have been investigated as potential therapeutic treatments for different human disorders. NR is a NAD precursor that has the ability to cross the plasma membrane and does not inhibit the activity of sirtuins and PARPs. Diet supplementation with NR was shown to induce the mitochondrial unfolded protein response and synthesis of prohibitin proteins, rejuvenate muscle stem cells in aged mice and in a mouse model of muscular dystrophy (Zhang et al. 2016). Similarly, administration of the NAD intermediate NMN, a product of the NAMPT reaction, has been shown to enhance NAD biosynthesis and to mitigate age-associated physiological decline in mice. Supplementation of NMN was shown to suppress age-associated body weight gain, enhance energy metabolism, promote physical activity, improve insulin sensitivity and the plasma lipid profile, and ameliorate reduced eye function and other pathophysiologies (Mills et al. 2016).

\subsubsection{NAD as Cofactor of Sirtuins and PARPs}

NAD is a critical cofactor for SIRTs, ARTs, and PARPs. Although these three classes of enzymes use NAD, they have distinct functional roles. In the next sections, the role of SIRT and PARP enzymes will be discussed and their regulation, which is mediated by NAD levels.

\subsubsection{Sirtuins}

Sirtuins are class III histone deacylases that remove acyl groups from lysine residues on proteins in a NAD-dependent manner (• Fig. 9.3). NAD is cleaved between nicotinamide and ADP-ribose, and the latter serves as an acyl acceptor, generating acyl-ADP-ribose.

In mammalian cells, there are seven sirtuins, SIRT1-7, which are located in distinct cellular compartments and can, therefore, coordinate cellular responses to

5 Progeroid syndromes are a group of rare genetic disorders that showed accelerating physiological ageing, such as hair loss, short stature, skin tightness, cardiovascular diseases and osteoporosis. 
caloric restriction. SIRT1, SIRT6, and SIRT7 are localized in the nucleus where they function to deacetylate histones, thereby affecting gene expression. SIRT2 is both a cytosolic and nuclear sirtuin and modulates cell cycle control. SIRT3, SIRT4, and SIRT5 are mitochondrial sirtuins and respond to caloric restriction by switching cells to favor mitochondrial oxidative metabolism (Chang and Guarente 2014).

Sirtuin function is intrinsically linked to cellular metabolism. Over the past two decades, accumulating evidence indicates that sirtuins are conserved, diet-sensitive, anti-aging proteins. Caloric restriction, a decrease in calorie intake (by 10 to $40 \%$ ) without malnutrition, has been shown to increase lifespan and healthspan in all organisms in which it has been tested. Moreover, as discussed above, NAD levels decline with aging, which would lead to a reduction in sirtuin activity. Initial studies in $S$. cerevisiae showed that the increased longevity induced by caloric restriction requires the activation of the sirtuin protein silent information regulator 2 (SIR2) by NAD (Lin et al. 2000). Since then, several other studies in flies and worms have confirmed the lifespan-extending effects of SIR2 homologs, underscoring the evolutionary significance of SIR2 in extending life span. Studies in mice also revealed a role of sirtuins in the regulation of aging and longevity in mammals. Transgenic mice overexpressing SIRT1 and SIRT6 exhibit phenotypes consistent with a delay in aging.

SIRT1 functions as a chromatin regulator by deacetylating specific histoneacetylated residues (e.g., H3K9ac, H3K14ac, and $\mathrm{H} 4 \mathrm{~K} 16 \mathrm{ac}$ ) but also transcription factors such as TP53, NF-kB, PGC-1a, and FOXO3a. SIRT1 protein abundance is relatively stable, but its deacetylase activity depends on NAMPT to generate NAD. An important example is provided by the regulation of SIRT1 activity in circadian clock regulation. SIRT1 is recruited to the Nampt promoter inhibiting the expression of NAMPT, which is a key enzyme of the NAD salvage pathway, and thus contributes to the circadian synthesis of its own coenzyme. Inhibition of Nampt promotes oscillation of the clock gene Per2 by releasing CLOCK:BMAL1 from suppression by SIRT1. In turn, the circadian transcription factor CLOCK binds to and up-regulates Nampt, thus completing a feedback loop involving NAMPT/NAD and SIRT1/CLOCK: BMAL1. Caloric restriction increases SIRT1 protein levels and induces neural activation in the dorsomedial and lateral hypothalamic nuclei. Increasing SIRT1 in the brain of transgenic mice enhances neural activity specifically in hypothalamic nuclei and promotes physical activity in response to different diet-restricting paradigms. However, moderate overexpression of SIRT1 in the whole organism was not sufficiently potent to affect longevity although old mice presented lower levels of DNA damage, decreased expression of the ageing-associated and cell cycle regulator gene $p 16 \operatorname{Ink} 4 \mathrm{a}^{6}$, a better general health, and fewer spontaneous carcinomas and sarcomas. Pharmacological interventions directed to increase SIRT1 activity have been found to slow the onset of aging and delay age-associated disease. This concept has been validated in mice treated with the natural polyphenol resveratrol, which activates SIRT1 and produces changes associated with longer lifespan, including increased insulin

6 Note that p16Ink4a is frequently silenced in cancer through DNA methylation (see $>$ Chap. 8 of Santoro) 
sensitivity, reduced insulin-like growth factor-1 (IGF-I) levels, increased AMPactivated protein kinase (AMPK) and peroxisome proliferator-activated receptor gamma coactivator 1-alpha (PGC-1 $\alpha$ ) activity, increased mitochondrial number, and improved motor function (Baur et al. 2006).

Like SIRT1, SIRT6 expression is also correlated with longevity. SIRT6 modulates telomeric chromatin through its association with telomeres and deacetylation of $\mathrm{H} 3 \mathrm{~K} 9 \mathrm{ac}$, which is required for the stable association of WRN, a factor that is mutated in the premature ageing disorder Werner syndrome. Accordingly, SIRT6depleted cells show premature cellular senescence and exhibit abnormal telomere structures that resemble defects observed in Werner syndrome.

The seven members of sirtuin family are considered potential targets for the treatment of human pathologies including neurodegenerative diseases, cardiovascular diseases, and cancer. The action of sirtuins as epigenetic players in the regulation of fundamental biological pathways has prompted increased efforts to discover small molecules able to modify sirtuin activity.

\subsubsection{PARPs}

Another group of enzymes that uses NAD as a substrate and regulates cellular NAD levels are the PARP enzymes (• Fig. 9.3). There are 17 PARP-related enzymes. PARP1 and PARP2 catalyze the polymerization of ADP-ribose units from NAD, resulting in the attachment of either linear or branched poly-(ADP-ribose) (PAR) polymers to itself or other target proteins.

Many early studies on PARPs and ADP-ribosylation were carried out with PARP1, the most ubiquitous and abundant PARP family member. PARP1 has been implicated in epigenetic and chromatin regulation. PARP1 can modulate chromatin structure and act as a transcriptional coregulator. Initial evidence of a role of PARP1 in the regulation of chromatin structure derives from pioneering studies reporting that PARylation of nucleosomes causes chromatin decondensation in vitro (Poirier et al. 1982). In Drosophila, activation of PARP1 promotes decondensation of chromatin in response to heat shock or other cellular signaling pathways (Tulin and Spradling 2003). Accordingly, histone H1 and PARP1 exhibit a reciprocal pattern of chromatin binding at many RNA polymerase II transcribed promoters. PARP1 was enriched whereas $\mathrm{H} 1$ was depleted at these promoters. Furthermore, PARP1 can act as coregulator by altering the function of components of the transcriptional machinery.

PARP1 is strongly activated by DNA damage and, through the production of long PAR chains, leads to consumption of a large amount of cellular NAD. PARP1 and SIRT1 have a similar Km for NAD (PARP1 50-97 $\mu \mathrm{M}$; SIRT1 94-96 $\mu \mathrm{M}$ ). Consequentially, upon PARP1 activation, the decrease of NAD concentration should lead to a decrease in SIRT1 activity. PARP1 KO mice phenocopied many aspects of SIRT1 activity, such as a higher mitochondrial content, increased energy expenditure, and protection against metabolic diseases. The pharmacologic inhibition of PARP increases NAD content and SIRT1 activity and enhances oxidative metabolism. Interestingly, the NAD boosting effects of PARP inhibition enhance the activity of SIRT1 in the nucleus, but not that of SIRT2 in the cytoplasm or SIRT3 in mitochondria. This finding suggests that NAD is independently regulated within different cellular compartments. However, the role of PARP1 in aging and longevity is still not clear. 
PARP inhibitors ${ }^{7}$ are currently used in the management of tumors with absent or dysfunctional BRCA genes such as breast and ovarian cancers.

\subsection{S-adenosylmethionine (SAM)}

$S$-adenosylmethionine (SAM) is the cofactor of DNA and histone methyltransferases and is the second most common enzymatic cofactor after ATP (• Fig. 9.5).

\subsubsection{Biosynthesis of SAM}

SAM plays major roles in epigenetics, biosynthetic processes including phosphatidylcholine, creatine, and polyamine synthesis, as well as sulfur metabolism (Ducker and Rabinowitz 2017). The methyl group of SAM serves as donor for transmethyltransferase reactions and is then converted into $S$-adenosylhomocysteine (SAH), which is a potent inhibitor of all methyltransferases (• Fig. 9.5).

The production of SAM and its control in the methylation of DNA and histones is a prominent example of how diet and nutrition can impact the epigenome. SAM is produced by one-carbon metabolism, which combines the folate cycle and the methionine cycle (• Fig. 9.6). One-carbon metabolism is an integrator of the nutrient status based on amino acids, glucose, and vitamins The products generated in these pathways are implicated in diverse cellular processes that include cellular biosynthesis, regulation of redox status, regulation of epigenetics through nucleic acid and protein methylation, and genome maintenance through the regulation of nucleotide pools.

Nutrients that fuel one-carbon metabolism are folate, serine, and glycine. Serine and glycine can be synthesized de novo. In contrast, folate can be synthesized by plants and most microorganisms whereas animals require dietary folate intake. In cells, folate is reduced into tetrahydrofolate (THF) by dihydrofolate reductase (DHFR). THF enters into the folate cycle and serves as scaffold that carries 1-carbon groups in a variety of reactions, including the production of methyl-THF (mTHF). The folate cycle is coupled to the methionine cycle through the demethylation of mTHF, which regenerate THF and allows methylation of homocysteine (hCYS) to produce methionine by methionine synthase and its cofactor vitamin B12. Methionine adenyltransferase (MAT) with ATP as cofactor converts methionine into SAM, which is then

7 The use of a PARP inhibitor in a BRCA-deficient cancers is the first example of the clinical application of the concept of synthetic lethality. BRCA1/2 genes produce proteins with an important role in repair of double strand DNA breaks (DSB) by homologous recombination (HR). While normal cells can repair DSBs through HR that is error free, loss of BRCA function forces cells to repair DSBs via non-homologous end joining NHEJ or the single-strand annealing sub-pathway of HR, both mechanisms being prone to error and genomic instability.

Ftnote_PARP1 and 2 are implicated in the repair of single-strand DNA break (SSB). PARP inhibitors trap the PARP complex and DNA replication stalls, converting SSBs into DSBs. When PARP inhibitors are given to cells with wild type BRCA1/2, the cells manage to repair DSBs by HR and survive. However, after administration of PARP inhibitors, tumor cells with mutated BRCA1/2 genes are unable to repair and undergo cell death. 
<smiles>C[S+](CC[C@H](N)C(=O)O)CC1O[C@H]2O[C@@H]1[C@@H](O)C2O</smiles>

S-adenosylmethionine (SAM)<smiles>Nc1ncnc2c1ncn2[C@@H]1O[C@H](C[SH+]CC[C@H](N)C(=O)[O-])[C@H](O)[C@H]1O</smiles>

S-adenosylhomocysteine (SAH)

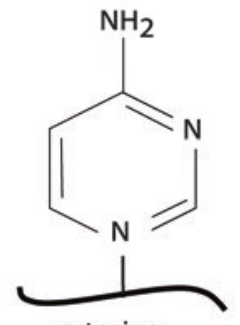

cytosine

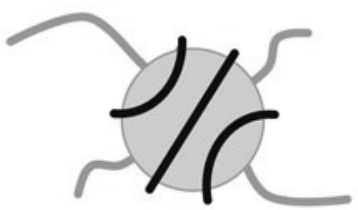

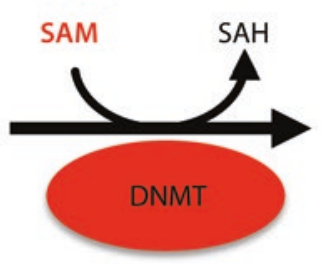

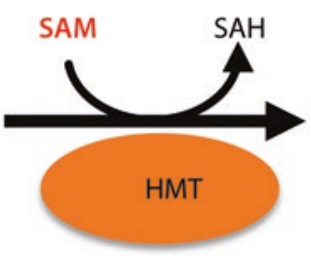

$\mathrm{H}{ }_{3} \mathrm{C}$

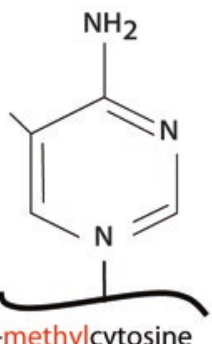

5-methylcytosine

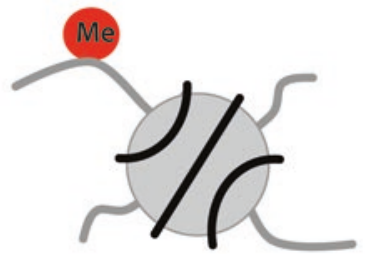

- Fig. 9.5 $S$-adenosylmethionine (SAM) is the cofactor of DNA and histone methyltransferases. SAM and $S$-adenosylhomocystein (SAH) are shown. SAM is the cofactor for DNA methyltransferase and histone methyltransferase. The methyl group of SAM serves as donor for trans-methyltransferase reactions. SAM is then converted into SAH, which is a potent inhibitor of all methyltransferases

demethylated to form SAH. Deadenylation of SAH by $S$-adenosyl homocysteine hydrolase $(\mathrm{SAHH})$ generates hCYS, resulting in a full turn of the methionine cycle.

\subsubsection{SAM as Cofactor of DNA and Histone Methyltransferases}

Enzymes that use SAM as cofactor are DNA methyltransferases (DNMTs), lysine methyltrasferases (KMTs), and peptidylarginine methyltransferases (PRMTs). The crosstalk between metabolism and chromatin is regulated by the kinetic properties of each enzyme and the physiological concentrations of the metabolites. This is particularly important for methyltransferases and acetyltransferases (see also Sect. 9.2.2), which respond to changes in metabolism due to low physiological concentrations of metabolic substrates that limit their enzymatic activities. 


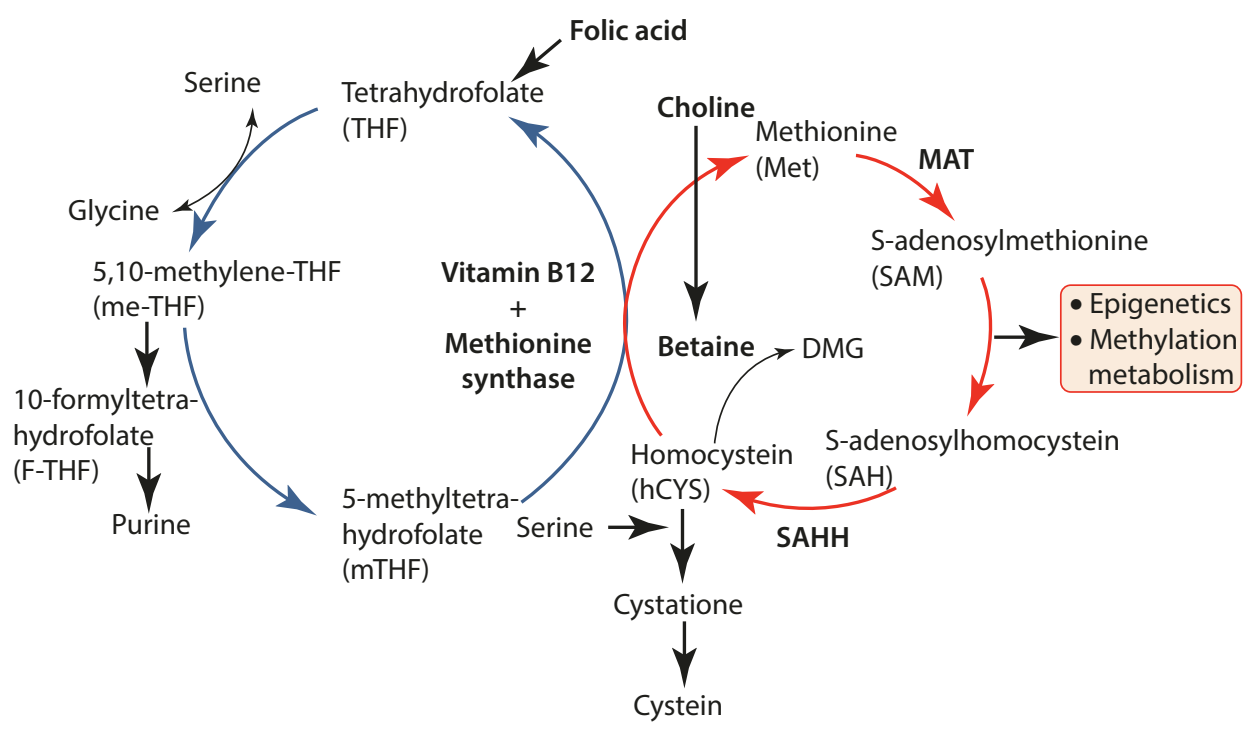

- Fig. 9.6 One-carbon metabolism. SAM is produced by one-carbon metabolism, which combines the folate cycle and the methionine cycle. Nutrients that fuel one-carbon metabolism are folate, serine, and glycine. DHFR reduces folate to THF, which enters into the folate cycle and serves as scaffold that carries 1-carbon groups in a variety of reactions, including the production of mTHF. The folate cycle is coupled to the methionine cycle through Vitamin B12 that serves as cofactor for the demethylation of $\mathrm{mTHF}$ and the production of methionine by methionine synthase. MAT enzyme converts methionine into SAM, which is then demethylated to form SAH. Deadenylation of SAH by SAHH generates hCYS, resulting in a full turn of the methionine cycle. Dihydrofolate reductase (DHFR); tetrahydrofolate (THF); methyl-THF (mTHF); homocysteine (hCYS); methionine adenyltransferase (MAT); $S$-adenosyl homocysteine hydrolase (SAHH)

Competition for available SAM may regulate the contrasting methylation events, such as methylation of histone $\mathrm{H} 3 \mathrm{~K} 4$ that is associated with transcription activity and methylation of DNA and histone $\mathrm{H} 3 \mathrm{~K} 9$ that are associated with transcriptional silencing. Thus, changes in one-carbon metabolism, and, hence, alterations in the levels of SAM, can have an impact on gene expression. For instance, low amounts of external methyl donor groups from dietary sources can reduce the concentrations of SAM and affect gene expression. An important example of how nutrients affect the epigenome is the agouti mouse model where the maternal methyl dietary content affects the coat color of the offspring ( $\bullet$ Fig. 9.7 and 9.8). The murine agouti gene encodes a paracrine signaling molecule that signals follicular melanocytes to switch from producing black eumelanin to yellow phaeomelanin. The agouti $(a)$ gene is transiently expressed in hair follicles resulting in a sub-apical yellow band on each black hair that causes the brown (agouti) coat color of wild-type mice. The Agouti viable yellow $\left(A^{\mathrm{vy}}\right)$ allele was first described in the early 1960s and resulted from the insertion of an intracisternal A particle (IAP) retrotransposon upstream of the transcription start site of the agouti gene. In mice carrying the $A^{\text {vy }}$ allele, a cryptic promoter in the proximal end of the IAP promotes constitutive, ectopic agouti transcription, resulting in the expression not only in hair follicles but in all cells. This leads to a yellow fur, as well as adult-onset obesity, diabetes, and tumorigenesis (• Fig.9.7). The activity of the $A^{\mathrm{vy}}$ allele is under the control of DNA methylation. In the case 

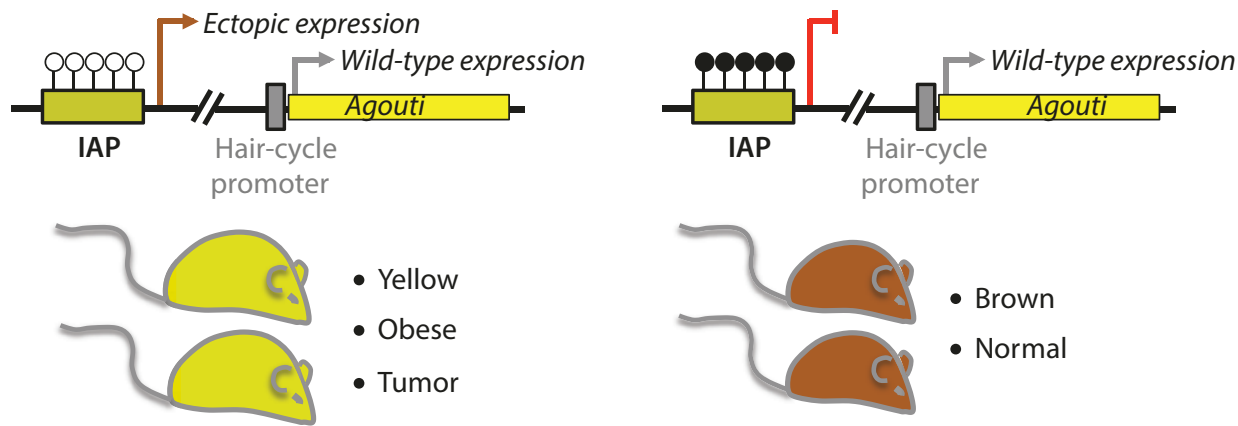

- Fig. 9.7 Regulation of Agouti viable yellow ( $A^{\text {vy }}$ ) by DNA methylation. The $A^{\text {vy }}$ allele results from the insertion of an intracisternal A particle (IAP) retrotransposon upstream of the transcription start site of the agouti gene. DNA methylation at the IAP represses the ectopic expression of the agouti gene, resulting in healthy mice with brown coat color

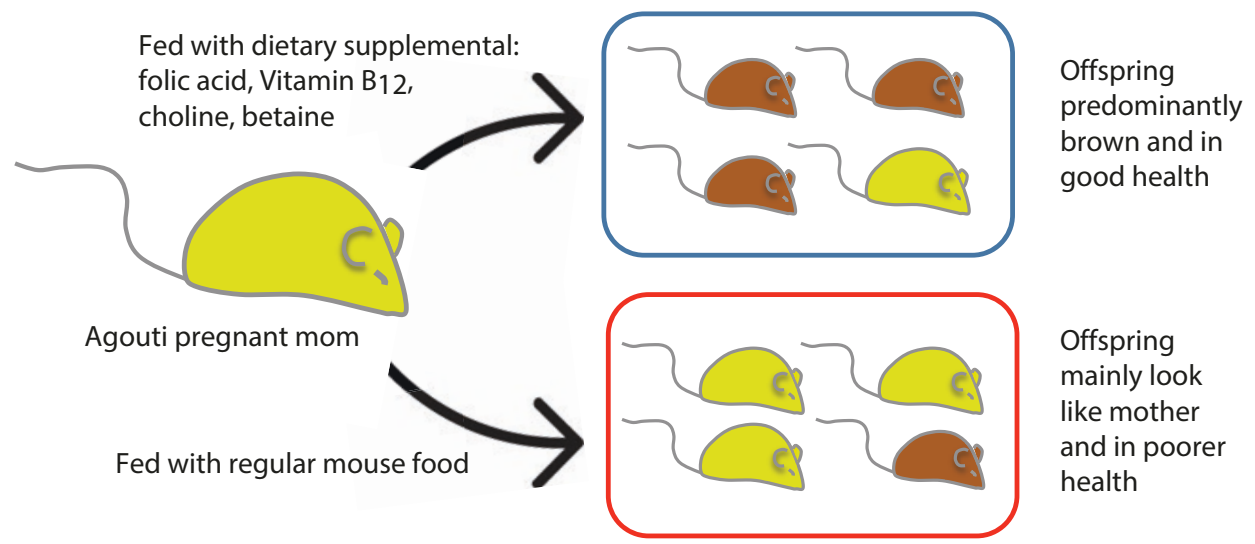

- Fig. 9.8 Effect of maternal dietary supplementation on the phenotype and epigenotype of $A^{\text {vy }}$ offspring. Maternal supplementation of $A^{\mathrm{vy}}$ mice with methyl donors (folate, betaine, vitamin $\mathrm{B}_{12}$, and choline) results in litters with a higher proportion of offspring with brown coats, whereas the offspring of mothers fed with a regular diet have a predominantly yellow coat color

of hypermethylation, the allele is silent and produces a wild-type, agouti-colored coat (termed pseudoagouti), whereas hypomethylation characterizes a transcriptionally active state that produces a completely yellow coat and increases the tendency towards obesity and cancer (Morgan et al. 1999) (• Fig. 9.7).

Thus, agouti and pseudoagouti mice are genetically identical but they differ in epigenetic state and phenotype. Remarkably, if a pregnant $A^{\mathrm{vy}}$ mouse receives dietary supplements such as vitamin B12, folic acid, choline, and betaine, fueling the onecarbon metabolism and leading to an increase in SAM production, the IAP in offspring will be methylated and agouti shows wild-type expression (• Fig. 9.8). These pups have a brown fur and no increased tendency towards obesity and cancer. Thus, environmental exposure or nutritional status can alter gene expression, especially during embryonic development, when the epigenome is established.

Chromatin-localized biosynthesis of metabolites has emerged as a process that modulates the expression of nearby genes by providing metabolites for epigenetic 
enzymes. This is of particular interest for methyltransferase reactions where interactions between SAM producers (metabolic enzymes) and SAM consumers (epigenetic enzymes) result in efficient chromatin modifications and an increased specificity for the correct modifications by limiting the use of SAM by enzymes with opposing outcomes (i.e. methylation of $\mathrm{H} 3 \mathrm{~K} 4$ versus methylation of H3K9). An example of this regulation is methionine adenosyltransferase (MAT), the enzyme that catalyzes the formation of SAM from methionine and ATP in one-carbon metabolism, which is a mitochondrial/cytosolic pathway. In mammals, MATII, one of the three distinct forms of MAT, localizes also to nuclei and interacts with the transcription factor MafK and H3K9 methyltransferases. The catalytic activity of MATII, which serves to supply SAM for methyltransferases, was necessary for $\mathrm{H} 3 \mathrm{~K} 9$ methylation and transcriptional repression of the heme oxygenase-1 gene (Katoh et al. 2011). The presence of microdomains for chromatin modifications, where SAM moieties for histone or DNA methyltransferases are immediately replenished after the modification, was also described for other epigenetic enzymes using, e.g., acetyl-CoA and NAD (see $>$ Sects. 9.2 and 9.3). Understanding how localized fluctuations in the levels of metabolites regulate chromatin states in space and time is a challenging question in the field of epigenetic research.

\subsection{Flavin Adenine Dinucleotide (FAD)}

Flavin adenine dinucleotide (FAD) is a cofactor of lysine demethylase 1 (KDM1 or LSD1) (• Fig. 9.9).

\subsubsection{Biosynthesis of FAD}

FAD is produced in mitochondria and the cytoplasm from riboflavin (vitamin B2), which can be found in many vegetables and meat. Riboflavin is phosphorylated by riboflavin kinase and ATP to generate riboflavin 5-phosphate, known as flavin
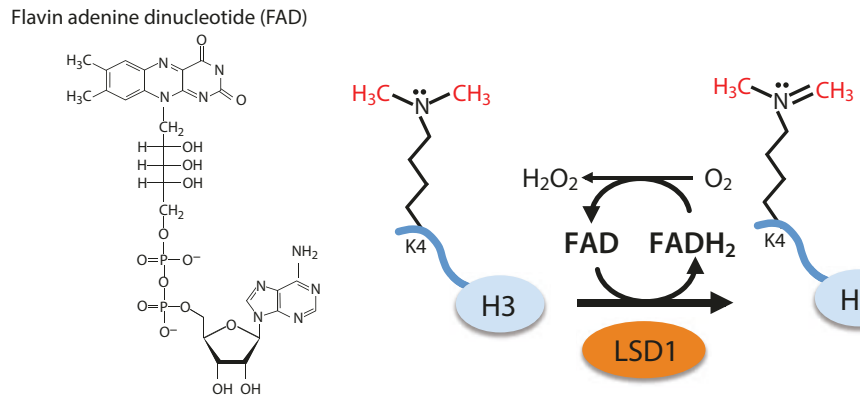

$\mathrm{H3}$

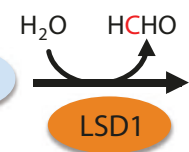

- Fig. 9.9 Flavin adenine dinucleotide (FAD) is a cofactor of lysine demethylase 1 (LSD1). The structure of FAD and its involvement in the histone demethylation mediated by LSD1 are shown. The reaction mediated by LSD1 requires protonation of the nitrogen to be demethylated, which limits LSD1 demethylating abilities to mono- and dimethylated lysines. LSD1-mediated demethylation generates formaldehyde and FADH2. Recycling of FAD occurs via oxidation of FADH2, which produces peroxide 
monucleotide (FMN), which is further converted to FAD by FMN adenyltransferase and ATP. Enzymes involved in FAD synthesis (FAD synthase) and hydrolysis (FAD pyrophosphatase) can also be found in the nucleus, providing a dynamic pool of nuclear FAD by balancing the ratio between its oxidized $\left(\mathrm{FAD}^{+}\right)$and reduced (FADH2) forms. The reduction of FAD to FADH2 is an essential reaction in the TCA cycle, where succinate dehydrogenase requires covalently bound FAD to catalyze the oxidation of succinate to fumarate. Finally, FADH2 can be re-oxidized by oxygen producing FAD and peroxide.

FAD acts as cofactor of flavoenzymes, which have dehydrogenase, oxidase, monooxygenase, or reductase activities. Flavoenzymes play crucial roles in bioenergetics, photochemistry, bioluminescence, redox homeostasis, chromatin remodeling, DNA repair, protein folding, apoptosis, and other physiologically relevant processes (Joosten and van Berkel 2007). Deficiency in FAD-dependent enzymes and/or impairment of flavin homeostasis in humans and animal models have been linked to several diseases, including cancer and neurological disorders.

\subsubsection{FAD as Cofactor of Lysine Demethylase 1 (LSD1)}

The majority of reported flavoenzymes localize to the mitochondria or cytoplasm. LSD1 is one of a few flavoproteins in the nucleus and is the only epigenetic enzyme that utilizes FAD as an essential cofactor for catalytic activity. LSD1 is a member of the flavin-containing amine oxidase family and represses transcription by removing the methyl group from mono-methylated and di-methylated H3K4 (Shi et al. 2004) (- Fig. 9.9). LSD1, in association with a nuclear receptor, is also involved in the demethylation of H3K9. Furthermore, demethylation by LSD1 was also reported for non-histone proteins such as p53. FAD-dependent monoamine oxidases typically catalyze the oxidation of amine-containing substrates, utilizing molecular oxygen as electron acceptor. The reaction mediated by LSD1 requires protonation of the nitrogen to be demethylated, which limits LSD1 demethylating abilities to mono- and dimethylated lysines. Of note is that LSD1-mediated demethylation generates formaldehyde and FADH2. Moreover, recycling of FAD occurs via oxidation of FADH2, which produces peroxide. The generation of formaldehyde and peroxide might potentially have deleterious effects when present near promoters. However, how cells deal with these products remains yet to be investigated.

The important function of LSD1 is evident by the embryonic lethality in nullmutant $K d m 1^{\mathrm{KO}}$ mice and LSD1-deficient ESCs displaying global DNA hypomethylation (Wang et al. 2009). The biological significance of FAD-dependent LSD1 activities in metabolic regulation have recently been supported by studies showing a crosstalk between energy metabolism and epigenetic gene regulation, e.g., by LSD1 repressing energy-expenditure genes such as $P G C 1 \alpha$ through $\mathrm{H} 3 \mathrm{~K} 4$ demethylation in adipocytes where excess energy is stored as triglycerides. Cellular FAD content remarkably increased during adipogenic differentiation while a reduction in FAD levels by downregulation of riboflavin kinase induced expression of most LSD1target genes. Moreover, transgenic overexpression of LSD1 in adipose tissue leads 

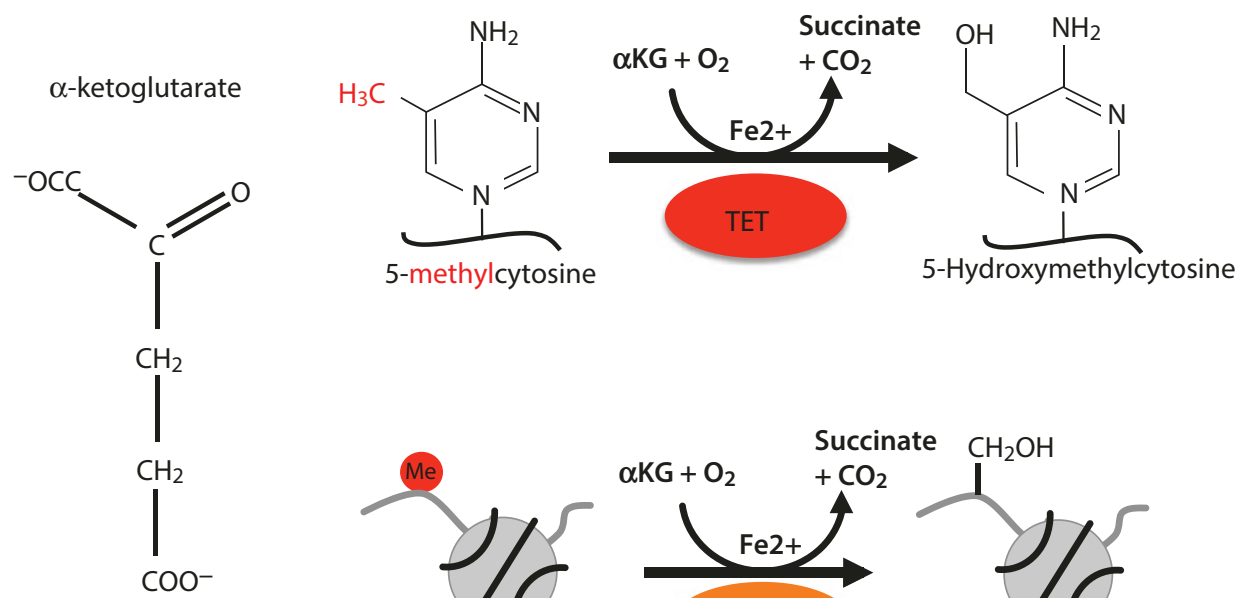

- Fig. 9.10 $\alpha$-ketoglutarate $(\alpha \mathrm{KG})$ is a co-substrate for dioxygenases. $\alpha \mathrm{KG}$ is a required cosubstrate for Jumonji C-family histone demethylases (KDMs) and TET enzymes, which participate in a multistep DNA demethylation process. TETs and KDMs require Fe(II) as a cofactor metal and $\alpha-\mathrm{KG}$ as a cosubstrate to catalyze the reactions in which one oxygen atom from molecular oxygen $\left(\mathrm{O}_{2}\right)$ is attached to a hydroxyl group in the substrate (hydroxylation) while the other is taken up by $\alpha \mathrm{KG}$, leading to the decarboxylation of $\alpha \mathrm{KG}$ and subsequent release of carbon dioxide $\left(\mathrm{CO}_{2}\right)$ and succinate

to increased browning ${ }^{8}$ by promoting mitochondrial activity, thus protecting against diet-induced obesity. Finally, LSD1 inhibitors lead to a shift from oxidative to glycolytic metabolism in brown adipose tissue, resulting in weight gain but improved glucose tolerance (Duteil et al. 2016). LSD1 inhibitors have entered in clinical trials to treat mixed lineage leukemia.

\section{$9.6 \alpha$-Ketoglutarate ( $\alpha$ KG)}

$\alpha$-ketoglutarate $(\alpha \mathrm{KG})$ is an intermediate of the TCA cycle and is a required cosubstrate for Jumonji domain-containing histone lysine demethylases (KDMs) and TET enzymes, which participate in a multi-step DNA demethylation process (• Fig.9.10).

\subsubsection{Biosynthesis of $\alpha$-Ketoglutarate}

Isocitrate dehydrogenases (IDH1, IDH2, IDH3) and glutamate dehydrogenase (GDH) are the enzymes producing $\alpha \mathrm{KG}$ (• Fig. 9.11).

IDH1 and IDH2 are nicotinamide adenine dinucleotide phosphate $\left(\mathrm{NADP}^{+}\right)$dependent enzymes whereas IDH3 is a NAD-dependent enzyme. IDH1 is localized to the cytoplasm and peroxisomes. IDH2 and IDH3 are localized to the mitochon-

8 Brown adipose tissue that has the function to dissipates energy to produce heat and has the potential to regulate body temperature by thermogenesis. 


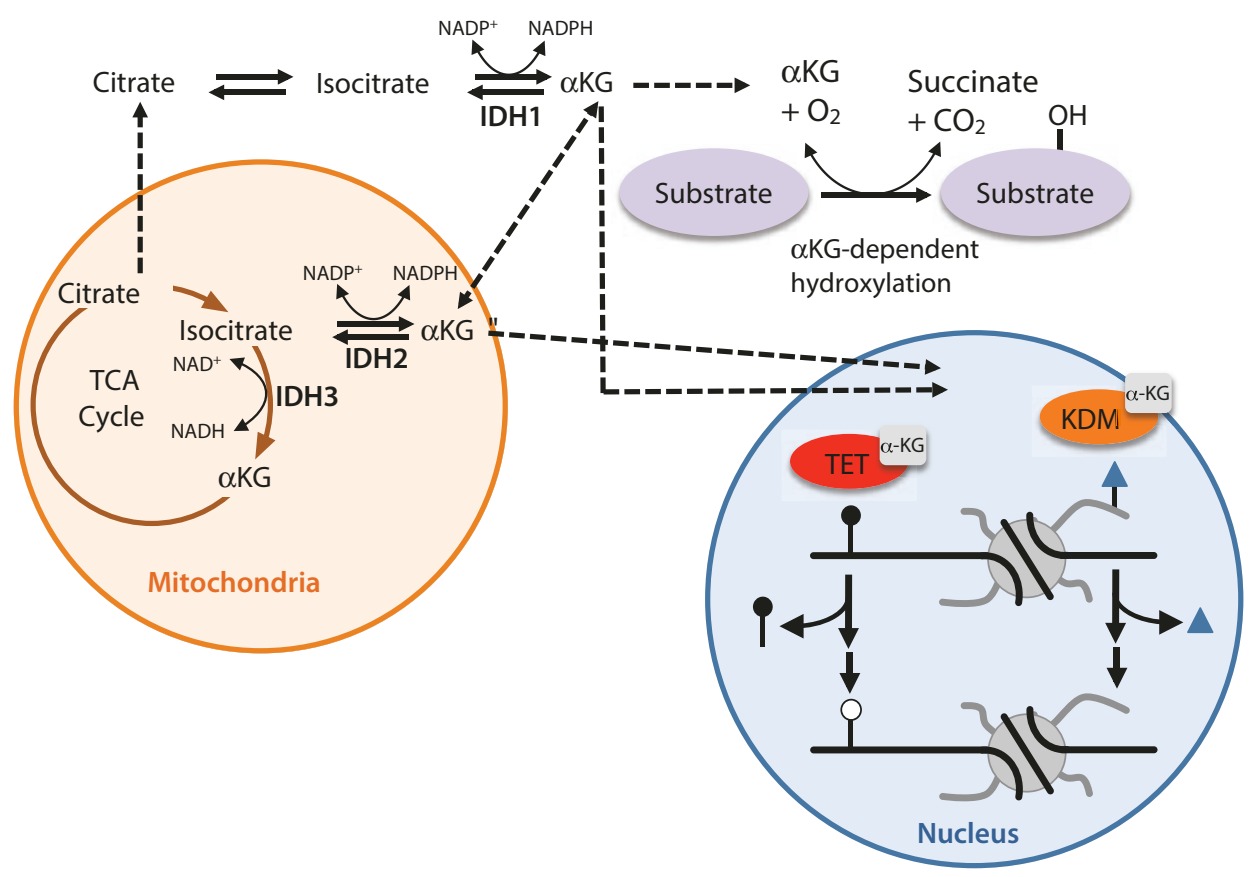

- Fig. 9.11 Biosynthesis of $\alpha$-ketoglutarate. Isocitrate dehydrogenases (IDH1, IDH2, IDH3) and glutamate dehydrogenase (GDH) are the enzymes producing $\alpha \mathrm{KG}$ from isocitrate. IDH1 is localized to the cytoplasm whereas IDH 2 and IDH3 are localized to the mitochondria. IDH1 and IDH2 are NADP ${ }^{+}$ dependent enzymes whereas IDH3 is a NAD-dependent enzyme

dria and play a key role in the regulation of the TCA cycle. IDH1 and IDH2 are bidirectional enzymes that can both produce and consume $\alpha \mathrm{KG}$ to meet cellular demands. $\alpha \mathrm{KG}$ is used for four separate pathways: TCA cycle, anaplerosis ${ }^{9}$, fatty acid synthesis, and protein and nucleic acid hydroxylation. $\alpha \mathrm{KG}$ is a rate-determining intermediate in the TCA cycle and has a crucial role in cellular energy metabolism. In the TCA cycle, $\alpha \mathrm{KG}$ is decarboxylated to succinyl-CoA and $\mathrm{CO}_{2}$ by the oxoglutarate dehydrogenase complex (OGDC), a key control point of the TCA cycle. $\alpha \mathrm{KG}$ also plays a major role in anaplerosis of the TCA cycle, a pathway that replenishes TCA intermediates from other biosynthetic pathways. In this case, $\alpha \mathrm{KG}$ serves as entry point into TCA cycle for several 5-carbon amino acids (Arg, Glu, Gln, His, and Pro) that, after the conversion into glutamate, undergo oxidative deamination catalyzed by $\mathrm{GDH}$ to form $\alpha \mathrm{KG}$ and NH3. $\alpha \mathrm{KG}$ can also be reduced by IDH1 and IDH 2 to isocitrate and then citrate. As discussed below, $\alpha \mathrm{KG}$ is used as a cosubstrate for multiple $\alpha$ KG-dependent dioxygenases that are involved in the hydroxylation of various proteins and nucleic acids.

9 Anaplerosis refers to metabolic pathways that replenish the TCA cycle intermediates. 


\subsection{2 $\alpha K G$ as Cofactor of TET-Family DNA Demethylases and Jumonji C-Family Histone Demethylases}

$\alpha \mathrm{KG}$ is used by several dioxygenases, which catalyze hydroxylation reactions on diverse substrates, including proteins and nucleic acids. TET-family DNA demethylases (TETs) and Jumonji C-family (JmjC) histone demethylases are $\mathrm{Fe}(\mathrm{II}) / \alpha \mathrm{KG}$ dependent dioxygenases that facilitate the removal of methyl groups from cytosine bases and histone residues, respectively (- Fig. 9.10). TET proteins mediate oxidation of $5 \mathrm{mC}$ to 5 -hydroxymethylcytosine $(5 \mathrm{hmC}), 5$-formylcytosine $(5 \mathrm{fC})$, and 5-carboxylcytosine $(5 \mathrm{caC})$, a reaction implicated in DNA demethylation (see book - Chap. 1 of Wutz). JmJC demethylases are a group of the histone lysine demethylases (KDMs). There are several different subfamilies of the JmjC KDMs that have been identified, including KDM2, KDM3, KDM4, KDM5, and KDM6. TETs and JmjC KDMs require Fe(II) as a cofactor metal and $\alpha \mathrm{KG}$ as a co-substrate to catalyze the reactions in which one oxygen atom from molecular oxygen $\left(\mathrm{O}_{2}\right)$ is attached to form a hydroxyl group in the substrate (hydroxylation) while the other is taken up by $\alpha \mathrm{KG}$, leading to the decarboxylation of $\alpha \mathrm{KG}$ and subsequent release of carbon dioxide $\left(\mathrm{CO}_{2}\right)$ and succinate (Hausinger 2004). The activity of TETs and JmjCs is competitively inhibited by TCA cycle intermediates such as succinate and fumarate.

Naive ESCs utilize both glucose and glutamine catabolism to maintain a high level of $\alpha \mathrm{KG}$. The elevated $\alpha \mathrm{KG}$ to succinate ratio in naive ESCs promotes histone and DNA demethylation and maintains pluripotency. Direct manipulation of the intracellular $\alpha \mathrm{KG} /$ succinate ratio is sufficient to regulate multiple chromatin modifications, including H3K27me3 and TET-dependent DNA demethylation, which contribute to the regulation of pluripotency-associated gene expression.

Initial genome-wide sequencing studies in patients with glioblastoma identified somatic mutations in $I D H 1$ and tumors without $I D H 1$ mutations often have mutations affecting IDH2 (• Fig. 9.12) (Parsons et al. 2008; Yan et al. 2009). Later, a similar mutation was also found in acute myelogenous leukemia (AML) and chondrosarcomas. Mutations in $I D H 1$ and $I D H 2$ are almost always in the binding site for isocitrate (Arg 132 for IDH1, Arg 172 for IDH2). However, mutant IDH1 and IDH2 are not simply inactive enzymes but, instead, possess a novel enzymatic activity that leads to the production of 2-hydroxyglutarate (2HG) (Dang et al. 2009). Accordingly, human malignant gliomas and AMLs harboring IDHI mutations contained elevated levels of $2 \mathrm{HG}$.

Evidence indicates that $2 \mathrm{HG}$ may act as an oncometabolite by competitive inhibition of $\alpha \mathrm{KG}$-dependent enzymes, including both JmjC KDMs and TETs (- Fig. 9.12). The structure of $2 \mathrm{HG}$ and $\alpha \mathrm{KG}$ is similar with the exception of the 2-ketone group in $\alpha \mathrm{KG}$ is replaced by a hydroxyl group in 2HG. Structural analysis showed that $2 \mathrm{HG}$ occupies the same space as $\alpha \mathrm{KG}$ does in the active site of histone demethylases and adopted a nearly identical orientation as $\alpha \mathrm{KG}$, thereby preventing the binding of $\alpha \mathrm{KG}$ to the active site of the enzymes.

In vitro studies revealed that $2 \mathrm{HG}$ strongly inhibits histone demethylases. The strongest inhibition was observed with KDM4A, which demethylates H3K9 and H3K36, followed by the H3K9/H3K36 demethylase KDM4C and the H3K36 demethylase KDM2A. These finding were supported by in vivo studies showing that human tumors expressing IDHI and IDH2 mutants had increased histone methylation at H3K4, H3K9, H3K27, and H3K79 (Lu et al. 2012). 


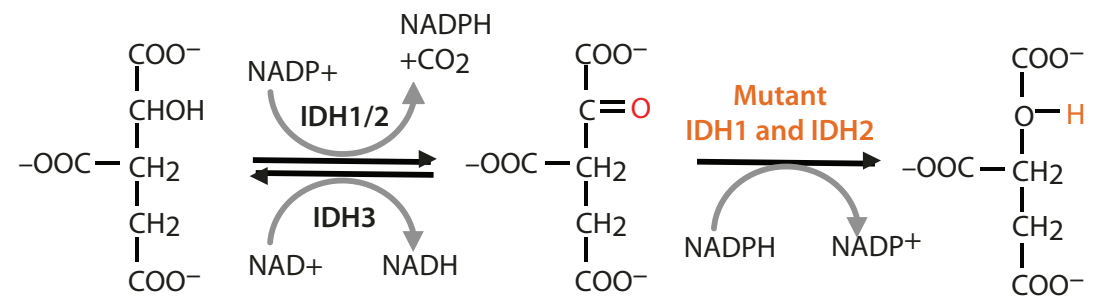

Isocitrate

5-methylcytosine

Q Unmethylated cytosine

Methylated lysine at histone $\alpha$-ketoglutarate

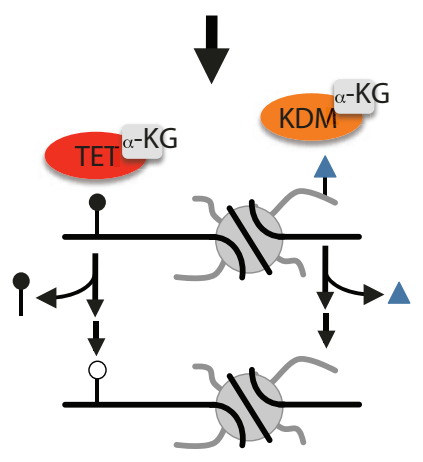

2-hydroxyglutarate (2HG)

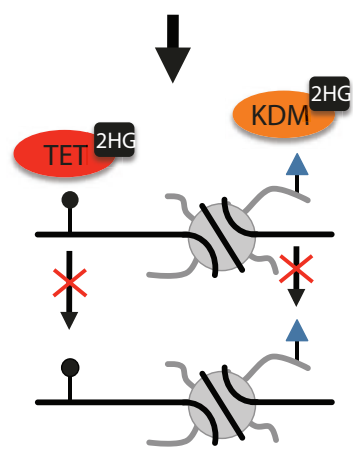

Hypermethylation at DNA and histones

- Fig. 9.12 Chemical reactions catalyzed by IDH enzymes and IDH1/2 mutants. IDH1 and IDH2 mutants possess a novel enzymatic activity that leads to the production of 2-hydroxyglutarate (2HG). $2 \mathrm{HG}$ acts as competitive inhibitor of $\alpha \mathrm{KG}$-dependent enzymes, including both KDMs and TETs, thereby causing hypermethylation at DNA and histones

The second major target of $I D H 1$ and $I D H 2$ mutations is the TET family. 2-HG inhibits TET activity and this inhibition could be overcome by the addition of $\alpha \mathrm{KG}(\mathrm{Xu}$ et al. 2011). Furthermore, glioblastomas expressing mutant IDHI display hypermethylation at a large number of loci, leading to what is known as the glioma-CpG island methylator phenotype. Remarkably, mutations affecting TET2 and IDH1 in AML (see - Chap. 8 of Santoro) occur in a mutually exclusive manner, suggesting that their biological effect is similar and that they have overlapping roles in AML pathogenesis.

\section{Take-Home Message}

- Most chromatin-modifying enzymes use metabolites that are central to cellular metabolism as cofactor

- The epigenome acts a sensor of the whole metabolic network and regulates gene expression accordingly.

- Environmental and physiological stimuli can alter metabolite concentration, which in turn might affect gene expression by modulating the activity of epigenetic and chromatin enzymes.

- Chromatin-localized biosynthesis of metabolites has emerged as a process that modulates the expression of nearby genes by providing metabolites that, as cofactors, activate epigenetic enzymes. 
- Specific maternal dietary treatments or environmental factors affect the phenotype of the offspring through epigenetic mechanism (see the agouti mouse).

- Chromatin and epigenetic pathways can be altered by oncometabolites, a class of metabolites whose quantity increase in tumors compared to normal cells. 2-HG, which is the result of $I D H 1$ or $I D H 2$ gene mutation in glioma and AML, impairs the function of dioxygenases requiring $\alpha \mathrm{KG}$ (e.g. TETs and KDMs) that are implicated in DNA and histone demethylation.

- Exercise and caloric restriction increase NAD levels and consequently activate Sirtuins that are implicated in the regulation of aging and longevity.

\section{References}

Baur JA, Pearson KJ, Price NL, Jamieson HA, Lerin C, Kalra A, Prabhu VV, Allard JS, Lopez-Lluch G, Lewis K, Pistell PJ, Poosala S, Becker KG, Boss O, Gwinn D, Wang M, Ramaswamy S, Fishbein KW, Spencer RG, Lakatta EG, Le Couteur D, Shaw RJ, Navas P, Puigserver P, Ingram DK, de Cabo R, Sinclair DA (2006) Resveratrol improves health and survival of mice on a high-calorie diet. Nature 444(7117):337-342. https://doi.org/10.1038/nature05354

Chang HC, Guarente L (2014) SIRT1 and other sirtuins in metabolism. Trends Endocrinol Metab 25(3):138-145. https://doi.org/10.1016/j.tem.2013.12.001

Dang L, White DW, Gross S, Bennett BD, Bittinger MA, Driggers EM, Fantin VR, Jang HG, Jin S, Keenan MC, Marks KM, Prins RM, Ward PS, Yen KE, Liau LM, Rabinowitz JD, Cantley LC, Thompson CB, Vander Heiden MG, Su SM (2009) Cancer-associated IDH1 mutations produce 2-hydroxyglutarate. Nature 462(7274):739-744. https://doi.org/10.1038/nature08617

Ducker GS, Rabinowitz JD (2017) One-carbon metabolism in health and disease. Cell Metab 25(1):2742. https://doi.org/10.1016/j.cmet.2016.08.009

Duteil D, Tosic M, Lausecker F, Nenseth HZ, Muller JM, Urban S, Willmann D, Petroll K, Messaddeq N, Arrigoni L, Manke T, Kornfeld JW, Bruning JC, Zagoriy V, Meret M, Dengjel J, Kanouni T, Schule R (2016) Lsd1 ablation triggers metabolic reprogramming of brown adipose tissue. Cell Rep 17(4):1008-1021. https://doi.org/10.1016/j.celrep.2016.09.053

Frederick DW, Loro E, Liu L, Davila A Jr, Chellappa K, Silverman IM, Quinn WJ 3rd, Gosai SJ, Tichy ED, Davis JG, Mourkioti F, Gregory BD, Dellinger RW, Redpath P, Migaud ME, Nakamaru-Ogiso E, Rabinowitz JD, Khurana TS, Baur JA (2016) Loss of NAD homeostasis leads to progressive and reversible degeneration of skeletal muscle. Cell Metab 24(2):269-282. https://doi.org/10.1016/j. cmet.2016.07.005

Hausinger RP (2004) FeII/alpha-ketoglutarate-dependent hydroxylases and related enzymes. Crit Rev Biochem Mol Biol 39(1):21-68. https://doi.org/10.1080/10409230490440541

Joosten V, van Berkel WJ (2007) Flavoenzymes. Curr Opin Chem Biol 11(2):195-202. https://doi. org/10.1016/j.cbpa.2007.01.010

Kaelin WG Jr, McKnight SL (2013) Influence of metabolism on epigenetics and disease. Cell 153(1):5669. https://doi.org/10.1016/j.cell.2013.03.004

Katoh Y, Ikura T, Hoshikawa Y, Tashiro S, Ito T, Ohta M, Kera Y, Noda T, Igarashi K (2011) Methionine adenosyltransferase II serves as a transcriptional corepressor of Maf oncoprotein. Mol Cell 41(5):554-566. https://doi.org/10.1016/j.molcel.2011.02.018

Lin SJ, Defossez PA, Guarente L (2000) Requirement of NAD and SIR2 for life-span extension by calorie restriction in Saccharomyces cerevisiae. Science 289(5487):2126-2128

Lu C, Ward PS, Kapoor GS, Rohle D, Turcan S, Abdel-Wahab O, Edwards CR, Khanin R, Figueroa ME, Melnick A, Wellen KE, O'Rourke DM, Berger SL, Chan TA, Levine RL, Mellinghoff IK, Thompson CB (2012) IDH mutation impairs histone demethylation and results in a block to cell differentiation. Nature 483(7390):474-478. https://doi.org/10.1038/nature10860

Mills KF, Yoshida S, Stein LR, Grozio A, Kubota S, Sasaki Y, Redpath P, Migaud ME, Apte RS, Uchida K, Yoshino J, Imai SI (2016) Long-term administration of nicotinamide mononucleotide mitigates age-associated physiological decline in mice. Cell Metab 24(6):795-806. https://doi. org/10.1016/j.cmet.2016.09.013 
Morgan HD, Sutherland HG, Martin DI, Whitelaw E (1999) Epigenetic inheritance at the agouti locus in the mouse. Nat Genet 23(3):314-318. https://doi.org/10.1038/15490

Moussaieff A, Rouleau M, Kitsberg D, Cohen M, Levy G, Barasch D, Nemirovski A, Shen-Orr S, Laevsky I, Amit M, Bomze D, Elena-Herrmann B, Scherf T, Nissim-Rafinia M, Kempa S, ItskovitzEldor J, Meshorer E, Aberdam D, Nahmias Y (2015) Glycolysis-mediated changes in acetyl-CoA and histone acetylation control the early differentiation of embryonic stem cells. Cell Metab 21(3):392-402. https://doi.org/10.1016/j.cmet.2015.02.002

Parsons DW, Jones S, Zhang X, Lin JC, Leary RJ, Angenendt P, Mankoo P, Carter H, Siu IM, Gallia GL, Olivi A, McLendon R, Rasheed BA, Keir S, Nikolskaya T, Nikolsky Y, Busam DA, Tekleab H, Diaz LA Jr, Hartigan J, Smith DR, Strausberg RL, Marie SK, Shinjo SM, Yan H, Riggins GJ, Bigner DD, Karchin R, Papadopoulos N, Parmigiani G, Vogelstein B, Velculescu VE, Kinzler KW (2008) An integrated genomic analysis of human glioblastoma multiforme. Science 321(5897):18071812. https://doi.org/10.1126/science. 1164382

Poirier GG, de Murcia G, Jongstra-Bilen J, Niedergang C, Mandel P (1982) Poly(ADP-ribosyl)ation of polynucleosomes causes relaxation of chromatin structure. Proc Natl Acad Sci U S A 79(11):34233427

Shi Y, Lan F, Matson C, Mulligan P, Whetstine JR, Cole PA, Casero RA (2004) Histone demethylation mediated by the nuclear amine oxidase homolog LSD1. Cell 119(7):941-953. https://doi.org/10.1016/j. cell.2004.12.012

Tulin A, Spradling A (2003) Chromatin loosening by poly(ADP)-ribose polymerase (PARP) at Drosophila puff loci. Science 299(5606):560-562. https://doi.org/10.1126/science.1078764. 299/5606/560 [pii]

Verdin E (2015) NAD(+) in aging, metabolism, and neurodegeneration. Science 350(6265):1208-1213. https://doi.org/10.1126/science.aac4854

Wang J, Hevi S, Kurash JK, Lei H, Gay F, Bajko J, Su H, Sun W, Chang H, Xu G, Gaudet F, Li E, Chen T (2009) The lysine demethylase LSD1 (KDM1) is required for maintenance of global DNA methylation. Nat Genet 41(1):125-129. https://doi.org/10.1038/ng.268

Wellen KE, Hatzivassiliou G, Sachdeva UM, Bui TV, Cross JR, Thompson CB (2009) ATP-citrate lyase links cellular metabolism to histone acetylation. Science 324(5930):1076-1080. https://doi. org/10.1126/science. 1164097

Xu W, Yang H, Liu Y, Yang Y, Wang P, Kim SH, Ito S, Yang C, Xiao MT, Liu LX, Jiang WQ, Liu J, Zhang JY, Wang B, Frye S, Zhang Y, Xu YH, Lei QY, Guan KL, Zhao SM, Xiong Y (2011) Oncometabolite 2-hydroxyglutarate is a competitive inhibitor of alpha-ketoglutarate-dependent dioxygenases. Cancer Cell 19(1):17-30. https://doi.org/10.1016/j.ccr.2010.12.014

Yan H, Parsons DW, Jin G, McLendon R, Rasheed BA, Yuan W, Kos I, Batinic-Haberle I, Jones S, Riggins GJ, Friedman H, Friedman A, Reardon D, Herndon J, Kinzler KW, Velculescu VE, Vogelstein B, Bigner DD (2009) IDH1 and IDH2 mutations in gliomas. N Engl J Med 360(8):765773. https://doi.org/10.1056/NEJMoa0808710

Zhang H, Ryu D, Wu Y, Gariani K, Wang X, Luan P, D'Amico D, Ropelle ER, Lutolf MP, Aebersold R, Schoonjans K, Menzies KJ, Auwerx J (2016) NAD(+) repletion improves mitochondrial and stem cell function and enhances life span in mice. Science 352(6292):1436-1443. https://doi. org/10.1126/science.aaf2693

Open Access This chapter is licensed under the terms of the Creative Commons Attribution 4.0 International License (http://creativecommons.org/licenses/by/4.0/), which permits use, sharing, adaptation, distribution and reproduction in any medium or format, as long as you give appropriate credit to the original author(s) and the source, provide a link to the Creative Commons license and indicate if changes were made.

The images or other third party material in this chapter are included in the chapter's Creative Commons license, unless indicated otherwise in a credit line to the material. If material is not included in the chapter's Creative Commons license and your intended use is not permitted by statutory regulation or exceeds the permitted use, you will need to obtain permission directly from the copyright holder.

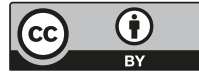

